\title{
524.
}

\section{ON THE DEFICIENCY OF CERTAIN SURFACES.}

[From the Mathematische Annalen, vol. III. (1871), pp. 526-529.]

IF a given point or curve is to be an ordinary or singular point or curve on a surface of the order $n$, this imposes on the surface a certain number of conditions, which number may be termed the "Postalation"; thus "Postulation of a given curve $q u \grave{a} i$-tuple curve on a surface $n$ " will denote the number of conditions to be satisfied by the surface in order that the given curve may be an $i$-tuple curve on the surface.

The "deficiency" (Flächengeschlecht) of a given surface of the order $n$ is

$=\frac{1}{6}(n-1)(n-2)(n-3)$ less deficiency-value of the several singularities; viz. as shown by $\operatorname{Dr}$ Noether, if the surface has a given $i$-tuple curve, the deficiency-value hereof is

$=$ Postulation of the curve quà $(i-1)$ tuple curve on a surface $n-4$;

and if the surface has an $i$-conical point, the deficiency-value hereof is

$=$ Postulation of the point quà $(i-2)$ conical point on a surface $n-4$; viz. this is $=\frac{1}{6} i(i-1)(i-2)$, and is thus independent of the order of the surface.

I remark that if the tangent-cone at the $i$-conical point has $\delta$ double lines and $\kappa$ cuspidal lines, then the deficiency-value is

$$
=\frac{1}{6} i(i-1)(i-2)+(i-2)(n-i-1)(\delta+\kappa) .
$$

In the case of a double or cuspidal curve $i$ is $=2$, and the deficiency-value is

$=$ Postulation of given curve $q u \grave{a}$ simple curve on a surface $n-4$;

and so for an ordinary conical point $i$ is $=2$, and the deficiency-value is $=0$ : results which were first obtained by Dr Clebsch. 
I found in this manner the expression for the deficiency of a surface $n$ having a double and cuspidal curve and the other singularities considered in my "Memoir on the Theory of Reciprocal Surfaces," Phil. Trans. vol. Clix. (1869), [411, Coll. Math. Papers, vol. vi. p. 356]; viz. this was

$$
D=\frac{1}{6}(n-1)(n-2)(n-3)-(n-3)(b+c)+\frac{1}{2}(q+r)+2 t+\frac{7}{2} \beta+\frac{5}{2} y+i-\frac{1}{8} \theta,
$$

where we have

$b$, order of double curve,

$q$, class of Do.,

$c$, order of cuspidal curve,

$r$, class of Do.,

$\beta$, number of intersections of the two curves, stationary points on $b$,

$y$, number of intersections, stationary points on $c$,

$i$, number of intersections, not stationary points on either curve,

$\theta$, number of certain singular points on $c$, the nature of which I do not completely understand; it is here taken to be $=0$.

Before going further I remark that

Postulation of right line quà $i$-tuple on surface $n$

$$
\begin{aligned}
& =\frac{1}{2} i(i+1) n-\frac{1}{6} i(i+1)(2 i-5), \\
& =\frac{1}{6} i(i+1)(3 n-2 i+5) .
\end{aligned}
$$

Whence if a surface $n$ has an $i$-tuple right line, the deficiency-value hereof is

or we have

$$
=\frac{1}{6} i(i-1)(3 n-2 i-5) \text {, }
$$

$$
\begin{aligned}
D & =\frac{1}{6}(n-1)(n-2)(n-3)-\frac{1}{6} i(i-1)(3 n-2 i-5) \\
& =\frac{1}{6}(i-n+1)(i-n+2)(2 i+n-3)
\end{aligned}
$$

so that $D=0$ if either $i=n-1$ or $i=n-2$; the former case is that of a scroll (skew surface) with a $(n-1)$ tuple right line, the latter that of a surface with a $(n-2)$ tuple line: whence (as shown by $\operatorname{Dr}$ Noether) such surface is rationally transformable into a plane.

For a surface of the order $n$ with an $i$-conical point where the tangent cone has $\delta$ double lines and $\kappa$ cuspidal lines, we have

$$
\begin{aligned}
D & =\frac{1}{6}(n-1)(n-2)(n-3)-\left\{\frac{1}{6} i(i-1)(i-2)+(i-2)(n-i-1)(\delta+\kappa)\right\} \\
& =\frac{1}{6}(n-i-1)\left\{n^{2}+n(i-5)+i^{2}-4 i+6-6(i-2)(\delta+\kappa)\right\} ;
\end{aligned}
$$

viz. for $i=n-1$ this is $D=0$ (in fact, a surface $n$ with a $(n-1)$ conical point is at once seen to be rationally transformable into a plane): and for $i=n$, that is, for a cone of the order $n$, we have

$$
D=-\frac{1}{2}(n-1)(n-2)+(n-2)(\delta+\kappa)-(n-3)(\delta+\kappa),
$$

where the last term $-(n-3)(\delta+\kappa)$ is added because in the present case the surface has the $\delta$ double lines and the $\kappa$ cuspidal lines.

$$
50-2
$$


The formula therefore gives

$$
D=-\frac{1}{2}(n-1)(n-2)+\delta+\kappa,
$$

viz. this is equal to the deficiency of the plane sections taken negatively.

I find that the same property exists first in the case of a scroll (skew surface) having only a double curve; and secondly in the case of a torse (developable surface) having a cuspidal curve with the ordinary singularities; and this being so there can I think be no doubt but that it is true for any scroll or torse whatever-viz. that for any ruled surface whatever the deficiency is equal to that of the plane section taken negatively.

First, for the scroll, we have

$$
D=\frac{1}{6}(n-1)(n-2)(n-3)-(n-3) b+\frac{1}{2} q+2 t,
$$

which should be

$$
=-\frac{1}{2}(n-1)(n-2)+b .
$$

Salmon's equations give in the case of a scroll

$$
\begin{aligned}
& 3 t=(n-4)\{3 b-n(n-2)\}, \\
& q=n(n-2)(n-5)-2(n-6) b,
\end{aligned}
$$

and with these values the relation is at orce verified.

Secondly, for the torse; changing the notation into that used for the singularities of the curve and torse, we have

$$
D=\frac{1}{6}(r-1)(r-2)(r-3)-(r-3)(x+m)+\frac{1}{2}(q+r)+2 t+\frac{7}{2} \beta+\frac{5}{2} \gamma+\alpha,
$$

which should be

$$
=-\frac{1}{2}(m-1)(m-2)+h+\beta .
$$

We have $q=r(n-3)-3 \alpha$, and substituting this value and expressing everything in terms of $r, m, n$ by means of the formulæ

$$
\begin{aligned}
& x=\frac{1}{2}\left(r^{2}-r-n-3 m\right), \\
& \alpha=m-3 r+3 n \\
& \beta=n-3 r+3 m, \\
& t=\frac{1}{6}\left\{r^{3}-3 r^{2}-58 r-3 r(n+3 m)+42 n+78 m\right\}, \\
& \gamma=r m+12 r-14 m-6 n, \\
& h=\frac{1}{2}\left(m^{2}-10 m-3 n+8 r\right),
\end{aligned}
$$

we have after all reductions

$$
D=-\frac{1}{2}(m+n)+r-1=-\frac{1}{2}(m-1)(m-2)+h+\beta .
$$


We have thus a class of surfaces of negative deficiency; viz. any rational transformation of a cone for which the plane section has a given (positive) deficiency produces such a surface: and I think it may be assumed conversely that a surface of negative deficiency is always the rational transformation of a cone for which the deficiency is equal to that of the surface taken with the reverse sign. As an instance, take a quintic surface having a nodal conic and two 3-conical (cubiconical) points (this of course implies that the line joining the two cubiconical points is a line on the surface); the formula for the deficiency is $(n=5, b=2, q=2, r=0, t=0)$

$$
D=\frac{1}{6}(n-1)(n-2)(n-3)-(n-3) b+\frac{1}{2} q+2 t-2,
$$

(viz. a term -1 for each of the cubiconical points)

$$
=4-4+1-2,=-1 .
$$

Such a surface can be obtained as the quadric inverse of a cubic cone; viz. taking for the vertex the point $x: y: z: w=\alpha: \beta: \gamma: \delta$ and the cone to pass through the point $x=0, y=0, z=0$, the equation of the cone is

$$
(\delta x-\alpha w, \quad \delta y-\beta w, \quad \delta z-\gamma w)^{3}=0,
$$

where $(\alpha, \beta, \gamma)^{3}=0$.

Taking $Q$ a quadric function $(x, y, z)^{2}$, the transformation in question consists in the change of $x, y, z, w$ into $x w, y w, z w, Q$; viz. the new equation, rejecting the factor $w$ which divides out, is

$$
\frac{1}{w}(\delta x w-\alpha Q, \quad \delta y w-\beta Q, \quad \delta z w-\gamma Q)^{3}=0,
$$

which is a quintic surface, having the two cubiconical points $x=0, y=0, z=0$ and $x: y: z: w=\alpha: \beta: \gamma: \frac{1}{\delta} Q_{0}$ (where $Q_{0}$ is the value of $Q$ on writing therein $\alpha, \beta, \gamma$ in place of $x, y, z)$ : and having the nodal conic $w=0, Q=0$.

Cambridge, 5 Jan. 1871. 